\title{
Thromboseprophylaxe \\ Niedermolekulare Heparine bei Patienten in der Onkologie
}

Bei Tumorpatienten ist das Thromboserisiko deutlich erhöht. Ursache dafür sind komplexe Wechselwirkungen zwischen Tumorzellen in der Blutbahn und Endothelzellen, die die Thrombose- und wahrscheinlich auch die Metastasenbildung begünstigen. Der Einsatz von Heparinen könnte sich daher in mehrfacher Hinsicht als sinnvoll erweisen.

Zwischen thrombotischen Ereignissen und Tumorerkrankungen besteht ein enger Zusammenhang. Bei Tumorpatienten treten venöse Thromboembolien (VTE) gehäuft als Komplikation auf. Aber auch bei Patienten mit idiopathischer VTE ist nicht selten ein Tumor die Ursache. Der Gefäßmediziner sollte dies insbesondere bei älteren VTE-Patienten immer bedenken und nach systemischen Ursachen forschen, so Prof. Dr. Michael Spannagl, München. Patienten, die von beiden Erkrankungen betroffen sind, haben eine sehr schlechte Prognose (Levitan $\mathrm{N}$ et al., 1999, Medicine 78: 285-291) und sollten antikoagulativ behandelt werden. Dabei sind niedermolekulare Heparine (NMH), wie z.B. Tinzaparin (innohep ${ }^{\circledR}$ ), Mittel der ersten Wahl und sollten entsprechend den Leitlinien der American Society of Clinical Oncology (ASCO) für mindestens sechs Monate in voller Dosierung gegeben werden (Lyman GH et al., 2007, J Clin Oncol 25: 5490-5505).

Dass die Patienten davon profitieren, wurde in einer randomisierten Studie zur Behandlung bei Patienten mit kleinzelligem Lungenkarzinom gezeigt: Das mediane Gesamtüberleben der Patientengruppe, die eine Chemotherapie plus NMH erhielt, betrug 13,0 Monate versus 8,0 Monate in der Patientengruppe, die die Chemotherapie plus Placebo erhielt ( $p=0,01$; Altinbas M et al., 2004, J Thromb Haemost 2: 1266-1271). In weiteren Studien war zumindest in der Subgruppe der Patienten mit guter Prognose ein längeres Gesamtüberleben durch die Gabe eines NMH zu erkennen (z.B. Klerk CP et al., 2005, J Clin Oncol 23: 2130-2135). Um die Effektivität von NMH konsequent zu überprüfen, wurde die Studie CATCH (Comparison of Acute Treatments in Cancer Haemostasis) initiiert, in der Tumorpatienten mit akuten Thrombosen für sechs Monate 175I.E./kg KG Tinzaparin oder einen Vitamin-K-Antagonisten erhalten.

\section{Anti-metastatische Wirkung durch NMH?}

Zwar sind die komplexen Beziehungen zwischen Tumoren und Thrombosen nicht vollständig aufgeklärt, bekannt ist aber, dass in der Blutbahn befindliche Tumorzellen durch Exprimierung des Tissue Factor die Endothelzellen aktivieren und die Gerinnungskaskade in Gang setzen können. Thrombin wird generiert, und es kommt u.a. zur Exozytose von Weibel-Palade-Körperchen und zur Freisetzung von P-Selektin sowie des Von-Willebrand-Faktors (VWF). Letzterer ist ein hochadhäsives Molekül, das normalerweise Plättchen bindet, erläuterte Prof.Dr.Stefan W.Schneider, Mannheim. In-vitro-Untersuchungen lassen vermuten, dass der VWF nicht nur Thrombozyten, sondern auch Tumorzellen binden kann, was letztendlich die Tumorextravasation und Metastasierung ermöglicht. Heparin kann diese Vorgänge unterbinden oder zumindest vermindern. Im Mausmodell wurden durch Heparin Metastasen verhindert, sodass Schneider hier Potenzial für ein antimetastatisches Medikament mit günstigem Nebenwirkungsprofil sieht. In der Studie TILT (Tinzaparin In Lung Tumours) soll untersucht werden, ob durch die zusätzliche Gabe eines NMH das Gesamt-

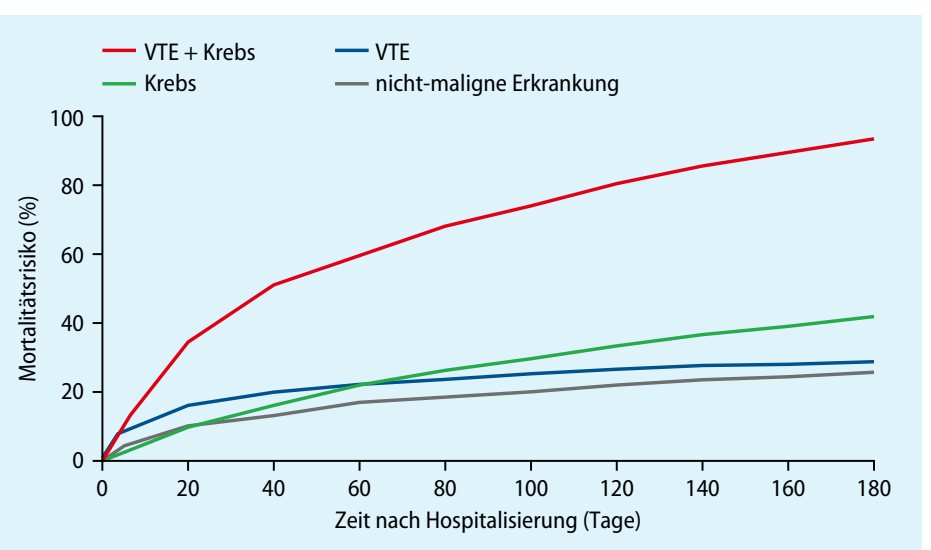

nach Levitan N et al., 1999, Medicine 78: 285-291

Höchstes Mortalitätsrisiko bei Tumorpatienten mit venösen Thromboembolien

überleben von Lungenkarzinompatienten verbessert werden kann (Meyer G et al., 2011, Rev Mal Respir 28: 654-659). CL

Quelle: Veranstaltung der LEO Pharma GmbH

\section{Fortgeschrittenes Pankreaskarzinom nab-Paclitaxel plus Gemcitabin}

Mit nab-Paclitaxel plus Gemcitabin kann bei fortgeschrittenem Pankreaskarzinom ein gutes Therapieergebnis erreicht werden, wie in einer Phase-I/II-Studie gezeigt wurde (von Hoff DD et al., 2011, J Clin Oncol 29:4548-4554).

Von 67 Patienten wurden 44 Patienten in den Phase-II-Teil der Studie eingeschlossen und mit $125 \mathrm{mg} / \mathrm{m}^{2} \mathrm{KOF}$ nab-Paclitaxel (Abraxane ${ }^{\circledR}$ ) plus $1.000 \mathrm{mg} / \mathrm{m}^{2}$ KOF Gemcitabin an den Tagen 1, 8 und 15 eines 28-Tages-Zyklus behandelt. Die Gesamtansprechrate betrug $48 \%$ und das mediane Gesamtüberleben (OS) 12,2 Monate. Die Auswertung speziell der Patienten, bei denen der SPARC (Secreted Protein Acidic and Rich in Cysteine)-Spiegel bestimmt wurde $(n=36)$, ergab dabei ein signifikant längeres OS bei hoher Expression des Proteins im Vergleich zu dem bei niedriger Expression (median 17,8 Monate versus 8,1 Monate; $p=0,0431$ ). Auch nach Adjustierung für verschiedene klinische Variablen blieb der SPARC-Spiegel ein signifikanter Prädiktor für das OS. Interessanterweise waren jedoch nur die SPARC-Spiegel im Stroma signifikant positiv mit dem OS korreliert $(p=0,013)$, nicht aber die Expression des Proteins in den Tumorzellen $(p=0,15)$. Dies ist überraschend, da nicht die SPARC-Expression im Tumor, sondern die im Stroma mit einer schlechten Prognose assoziiert ist. Die Ergebnisse der Studie sollen nun in einer Phase-III-Studie verifiziert werden. Quelle: Information der Celgene GmbH 